\title{
Attenuation of Postischemic Genomic Alteration by Mesenchymal Stem Cells: a Microarray Study
}

\author{
Chunggab Choi, ${ }^{1,6}$, Seung-Hun $\mathrm{Oh}^{2,6}$, Jeong-Eun Noh', Yong-Woo Jeong ${ }^{1}$, Soonhag Kim ${ }^{3,4}$, Jung Jae Ko', \\ Ok-Joon Kim ${ }^{2, *}$, and Jihwan Song ${ }^{1,5, *}$
}

\begin{abstract}
Intravenous administration of mesenchymal stem cells (IVMSC) protects the ischemic rat brain in a stroke model, but the molecular mechanism underlying its therapeutic effect is unclear. We compared genomic profiles using the mRNA microarray technique in a rodent stroke model. Rats were treated with $1 \times 10^{6}$ IV-MSC or saline (sham group) $2 \mathrm{~h}$ after transient middle cerebral artery occlusion (MCAO). MRNA microarray was conducted $72 h$ after MCAo using brain tissue from normal rats (normal group) and the sham and MSC groups. Predicted pathway analysis was performed in differentially expressed genes (DEGs), and functional tests and immunohistochemistry for inflammation-related proteins were performed. We identified 857 DEGs between the sham and normal groups, with the majority of them (88.7\%) upregulated in sham group. Predicted pathway analysis revealed that cerebral ischemia activated 10 signaling pathways mainly related to inflammation and cell cycle. IV-MSC attenuated the numbers of dysregulated genes in cerebral ischemia (118 DEGs between the MSC and normal groups). In addition, a total of 218 transcripts were differentially expressed between the MSC and sham groups, and most of them (175/218 DEGs, 80.2\%) were downregulated in the MSC group. IV-MSC reduced the number of Iba-1 $1^{+}$cells in the periinfarct area, reduced the overall infarct size, and improved functional deficits in MCAo rats. In conclusion, transcriptome analysis revealed that IV-MSC attenuated postischemic genomic alterations in the ischemic brain. Amelioration of dysregulated inflammation- and cell cycle-related gene expression in the host brain is one of the molecular mechanisms of IV-MSC therapy for cerebral ischemia.
\end{abstract}

\footnotetext{
${ }^{1}$ Department of Biomedical Science, CHA University, Seongnam 463400 , Korea, ${ }^{2}$ Department of Neurology, CHA Bundang Medical Center, CHA University, Seongnam 463-712, Korea, ${ }^{3}$ Institute for Bio-Medical Convergence, College of Medicine, Catholic Kwandong University, Gangneung 25601, Korea, ${ }^{4}$ Catholic Kwandong University International St. Mary's Hospital, Incheon 22711, Korea, ${ }^{5} \mathrm{CHA}$ Stem Cell Institute, CHA University, Seongnam 463-400, Korea, ${ }^{6}$ These authors contributed equally to this work.

*Correspondence: jsong@cha.ac.kr (JS); okjun77@ cha.ac.kr (OJK)
}

Received 24 November, 2015; revised 22 December, 2015; accepted 23 December, 2015; published online 29 February, 2016

Keywords: inflammation, mesenchymal stem cells, microarray, stroke, transcriptome

\section{INTRODUCTION}

The pathomechanisms of ischemic stroke are complex. The blockade of cerebral blood flow and subsequent hypoxia leads to neuronal death through several mechanisms including energy failure, neuronal excitotoxicity, neuroinflammation, and apoptosis (Eltzschig and Eckle, 2011; ladecola and Anrather, 2011). During cerebral ischemia, the expression of numerous genes, which are those involved in cell cycle, immune response and cell survival are dysregulated following neuronal injury, resulting in neuronal death and subsequent functional deficits (Slevin et al., 2005). The recent development of transcriptome analysis with mRNA microarrays had shed light on the molecular mechanisms of acute cerebral ischemia (Lu et al., 2003; Ramos-Cejudo et al., 2012; Tang et al., 2002). In addition to studying disease pathogeneses, transcriptome analysis is a powerful tool to elucidate the molecular mechanisms of candidate therapeutics (Liesz et al., 2014).

Mesenchymal stem cells (MSCs) are a promising cell source for the treatment of ischemic stroke. Intravenous administration of MSCs (IV-MSC) is safe and effective in animal stroke models (Chen et al., 2001; Deng et al., 2010; Ikegame et al., 2011; Kim et al., 2007; Zhang et al., 2011) and clinical cases (Bang et al., 2005; Honmou et al., 2011; Lee et al., 2010). Despite limited migration and a low engraftment rate to the injury site (Walczak et al., 2008; Zhang et al., 2011), systemic MSC administration can induce functional improvement in cerebral ischemia by paracrine mechanisms.

MSCs exert a strong immunomodulatory effect by reducing cytokine and chemokine activation and stimulating the production of growth factors that help the brain recover from tissue damage (Liu et al., 2014). However, how MSC treatment alters gene expression in the host brain is unclear. To understand the effect of IV-MSC in cerebral ischemia, it is necessary to evaluate the whole-genome expression pattern in the host brain. In the present study, we performed transcriptome analyses with mRNA microarray in a rodent stroke model to investigate the therapeutic mechanisms of IV-MSC in cerebral ischemia.

\section{MATERIALS AND METHODS}

\section{Ethics statement}

The cells used in this study were derived from the umbilical cord (UC) of a single healthy donor at CHA Bundang Medical Center (Korea) with informed consent. This study was performed following the approval of an Institutional Review Board 
from CHA Bundang Medical Center for the use of human UC resource (IRB no.: BD2013-004D). All of the experimental animals were manipulated in accordance with the Institutional Animal Care and Use Committee of CHA University (IACUC No.: 130006).

\section{MSC preparation}

To isolate MSCs, the umbilical vessels were removed, and Wharton's jelly was sliced into 1-5 mm explants that were subsequently cultured in $\alpha$-minimal essential medium ( $\alpha-M E M$; Invitrogen, USA) supplemented with $10 \%$ fetal bovine serum (FBS; Hyclone, USA), fibroblast growth factor 4 (FGF4), and heparin on culture plates. The medium was changed every 3 days, and MSC cell populations appeared as outgrowths from the UC fragments on day 6 . After 15 days, the UC fragments were discarded, and the cells were passaged using TrypLE (Invitrogen) and expanded until they reached subconfluence $(80-90 \%)$. The cells were incubated under hypoxic conditions $\left(3 \% \mathrm{O}_{2}, 5 \% \mathrm{CO}_{2}\right.$, and $\left.37^{\circ} \mathrm{C}\right)$. In the present study, cells were used in experiments at passage 7 . To identify MSC immunophenotypes, we performed fluorescence-activated cell sorting analysis as described previously (Kim et al., 2013). The cells expressed high levels of CD44 (99.94 $\pm 0.01 \%)$, CD73 (100 \pm $0.01 \%)$, CD90 (99.85 $\pm 0.06 \%)$, and CD105 (99.96 $\pm 0.03 \%)$ but negligible expressions of CD31 (1.43 $\pm 0.16 \%)$, CD34 (0.48 $\pm 0.06 \%)$, CD45 $(1.63 \pm 0.25 \%)$, and human leukocyte antigen (HLA)-DR $(0.86 \pm 0.10 \%)$. The cells were differentiated into adipocytes, osteocytes, and chondrocytes according to a previously described method (Kim et al., 2013).

\section{Rodent stroke model and IV-MSC treatment}

Male Sprague-Dawley rats weighing 270-300 g were subjected to a 90-min middle cerebral artery occlusion (MCAo) animal model (Longa et al., 1989). Two hours after reperfusion, a total of $1 \times 10^{6}$ MSCs mixed with $500 \mu \mathrm{l}$ saline was infused into the tail vein over 5 minutes (MSC group). The sham group only received saline with the same volume. No profound bleeding occurred during treatment, and the vital signs of all rats remained stable. The total mortality rates were evaluated for 4 weeks after treatment.

\section{Whole transcriptome analysis}

At $72 \mathrm{~h}$ after MCAo, the ipsilateral hemisphere subjected to MCAo was used for mRNA microarray. RNA was isolated as quickly as possible (within $20 \mathrm{~min}$ ) from rats without MCAo (normal group, $\mathrm{n}=6$ ), rats treated with $1 \times 10^{6} \mathrm{IV}-\mathrm{MSC}$ (MSC group, $n=7$ ), and rats treated with saline (sham group, $n=5$ ) by homogenization with TRIzol ${ }^{\circledR}$ Reagent (Gibco) and RNeasy columns (Qiagen, Germany). For RNA quality, the optical density (OD) cutoff at a 260/280 nm ratio was $>1.8$ using an Agilent 2100 Bioanalyzer (Agilent Technologies, USA). One sample from the normal group and two from the sham group were discarded because of low total RNA volume and low RNA quality, respectively. Afterwards, microarray experiments were conducted using samples from five rats in the normal group, five rats in the sham group, and six rats in the MSC group. RNA labeling and purification were performed, and the samples were hybridized to Agilent rat mRNA microarray chips (SurePrint G3 Rat Gene Expression 8x60k, Agilent Technologies) according to the manufacturer's instructions. Each array was scanned using the Agilent Technologies G2600D SG12494263. Array data-exporting processing and analyses were performed using Agilent Feature Extraction software (v100.0.1.1). The data were filtered by flag $A$, log transformation, and quantile normalization. The data from the three groups were analyzed using one-way variance of analysis (ANOVA) with correction of false discovery rate (FDR), and data with corrected p-values < 0.05 (FDR- $p<$ 0.05) were selected. Post hoc analysis using Tukey's honest significant difference (HSD) test was conducted for pair-wise comparison. A differentially expressed gene (DEG) was defined as a fold difference (FD) $\geq 1.5$ and corrected $p$ value $(p)$ $<0.05$. Predicted pathway analysis for the significant probe list was performed using DAVID Bioinformatics Resource ver. 6.7 (http://david.abcc.ncifcrf.gov) by inputting DEGs from the microarray data. The significantly predicted signaling pathway was determined based on $p<0.05$ after correction of FDR. All data analyses and visualization of DEGs were conducted using R 3.0.1 (www.r-project.org).

\section{Immunohistochemistry}

Rats were transcardially perfused with heparinized saline. Brains were fixed overnight in $4 \%$ formalin at room temperature and then transferred to $30 \%$ sucrose with shaking for 48 to $72 \mathrm{~h}$ at $4^{\circ} \mathrm{C}$. The brains were frozen in O.C.T. compound (Sakura Finetek, Japan). Double immunofluorescence labeling was performed on free-floating $40 \mu \mathrm{m}$-thick sections. Sections were incubated in blocking solution ( $5 \%$ normal horse serum, Vector Laboratories, USA) in phosphate-buffered saline (PBS) containing $0.3 \%$ Triton X-100 (Sigma, USA) for $1 \mathrm{~h}$ at room temperature. For evaluation of microglial activation, immunohistochemistry was performed using anti-lba1 antibody (rabbit polyclonal, 1:250; Wako Pure Chemical Industries, Japan). After washing in PBS, the brain sections were incubated with fluorescence-conjugated secondary antibodies: goat anti-mouse IgG-conjugated Alexa 488 and 555, goat anti-rabbit IgG-conjugated Alexa 488 and 555 (1:200; Molecular Probes, USA) for 2 h. All sections were counterstained with the nuclear marker 4', 6-diamidine-2'-phenylindole dihydrochloride (DAPI, Roche, USA).

\section{Functional tests and infarct size measurement}

Functional tests were conducted by independent investigators blinded to the treatment groups. The modified neurological severity score (mNSS) was calculated as previously described (Chang et al., 2013). For functional tests, rats were trained under the same conditions three times a day for 3 consecutive days prior to MCAo induction to reduce inter-animal variations. Infarct size was compared $72 \mathrm{~h}$ after MCAo using 2,3,5 triphenyltetrazolium chloride (TTC) staining. The brain was removed, and $1 \mathrm{~mm}$ was cut from the frontal tip and immersed in a $2 \%$ TTC solution. Stained slices were fixed in phosphatebuffered $4 \%$ paraformaldehyde. The ischemic and total hemispheric areas of each section were traced and measured, and the values were summed from six serial coronal sections per brain. We estimated infarct size as a percentage of the intact contralateral hemisphere with the following equation: estimated infarct size $(\%)=(1$ - [area of remaining ipsilateral hemisphere/area of intact contralateral hemisphere] $) \times 100$. The areas of interest were measured using ImageJ software (National Institutes of Health, USA), and values were summed from six serial coronal sections per brain. Ischemic volumes were measured by independent investigators blinded to the treatments.

\section{Statistical analysis}

Statistical analysis was performed using the Statistical Analysis System program (Enterprise 4.1; SAS Korea) and MedCalc statistical software (MedCalc software, ver. 11.6., Mariakerke, Belgium). Statistically significant differences between two groups 
A

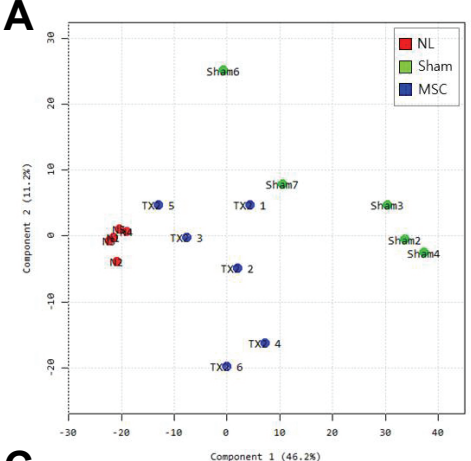

C

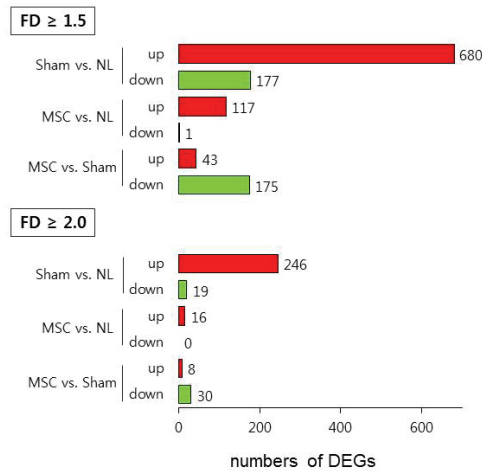

B

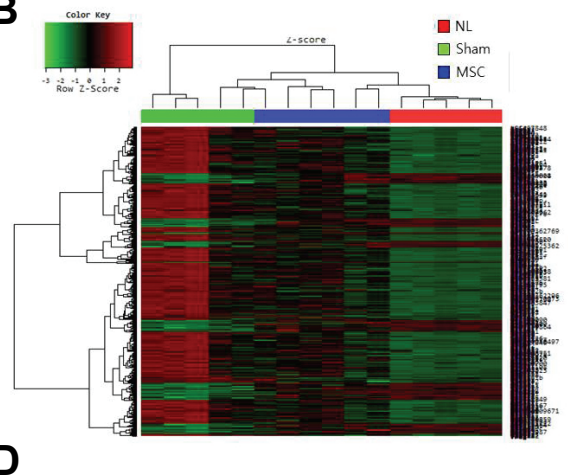

FD

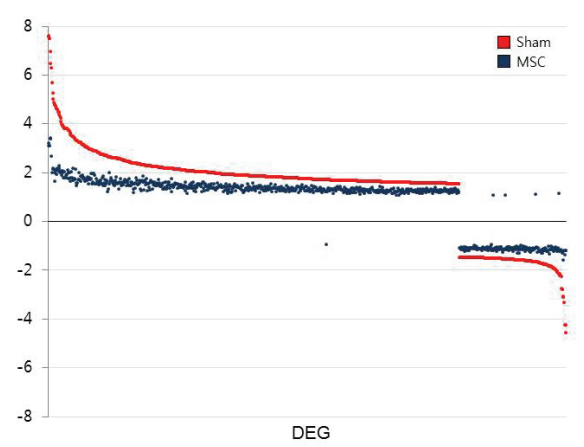

Fig. 1. Genomic profiling in the rat brain after cerebral ischemia and IV-MSC treatment. (A) 2D-multidimensional scaling (MDS) plot in the normal (NL group, red circles), MCAo (sham group, green circles), and IV-MSC (MSC group, blue circles) rats. (B) Heat-map of differentially expressed genes (DEGs) in the normal (NL), sham, and MSC groups. (C) Numbers of DEGs according to the threshold of fold difference (FD $\geq 1.5$ or $F D \geq 2.0$ ) in expressions between the normal (NL), sham, and MSC groups. (D) Dot-plot showing the FD of the expression of individual DEGs $(n=857)$ in the sham (red dots) and MSC (dark-blue dots) groups compared to the normal group. for infarct size and immunohistochemical data were analyzed with Mann-Whitney $U$ tests. Functional tests were analyzed with mixed ANOVAs. $p<0.05$ was considered significant, and all values are presented as means \pm standard error of mean (SEM). The statistical analysis of microarray data was previously described in the whole transcriptome analysis section.

\section{RESULTS}

Genomic profiling changes in the ischemic brain after IVMSC treatment

We performed mRNA microarray-based whole transcriptome analyses on brain tissue $72 \mathrm{~h}$ after MCAo and compared normal $(n=5)$, sham $(n=5)$, and MSC $(n=6)$ groups. The 2Dmultidimensional scaling (MDS) plot (Fig. 1A) shows a homogeneous genomic pattern in the normal group. Conversely, the sham and MSC groups exhibited wider plot distributions compared to the normal group, but a distinct plot distribution was detected between the sham and MSC groups. Of the 30,367 probes in the microarray, 19,035 were detected from the brain tissues used in this study. Among these, 5,414 probes were selected for FDR- $p<0.05$. After further selection of genes with $\mathrm{FD} \geq 1.5$ found at least in one tested group, we selected a total of 857 probes as DEGs (FD $\geq 1.5$, FDR- $p<0.05$ ) (Fig. 1B). In a post hoc comparison between the sham and normal groups, a total of 857 DEGs were detected (Fig. 1C). In the sham group, most DEGs (680 of 857 transcripts, $79.3 \%$ ) were upregulated compared to the normal group. The number of DEGs between the MSC and normal groups (118 DEGs) was much smaller than those between the sham and normal groups (857 DEGs) (Fig. 1C). The DEGs among the three groups are listed in Supplementary Table S1. In a post hoc comparison between the
MSC and sham groups, a total of 218 transcripts were differentially expressed, and most of them (175/218 DEGs, 80.2\%) were downregulated in the MSC group compared to the sham group (Fig. 1C). The list of DEGs between the MSC and sham groups is presented in Supplementary Table S2. A dot-plot of gene expression for all DEGs shows that the extent of gene expression in the MSC group was relatively low compared to the sham group (Fig. 1D). For DEGs with FD $\geq 2$ between the MSC and sham groups, the expressions of $30 / 38$ (78.9\%) transcripts in the MSC group were relatively reduced compared to those in the sham group (Fig. 2). These findings indicate that the expressions of numerous genes are changed (most are upregulated) in the host brain after cerebral ischemia, and IVMSC attenuates the extent of postischemic genomic alterations in the ischemic brain.

We next performed predicted pathway analysis with DAVID software by inputting 857 DEGs between the normal and sham groups (Supplementary Table S1) and 218 DEGs between the MSC and sham groups (Supplementary Table S2). For the first comparison, 10 pathways were predicted (corrected $p<0.05$, Table 1). Most were related to inflammation, such as antigenmediated immune response, pattern recognition immune response, and cytokine and chemokine signaling. In the comparison between the MSC and sham groups, three pathways were predicted: cell cycle, systemic lupus erythematosus (SLE), and Fc-gamma receptor (FcyR)-mediated phagocytosis (corrected $p$ $<0.05$, Table 2). Interestingly, most of the genes that were upregulated in the sham group compared to the normal group were downregulated in the MSC group compared to sham groups. These findings suggest that cerebral ischemia activates multiple signaling pathways mainly involved in inflammation and cell cycle-related signaling pathways, and IV-MSC partially 


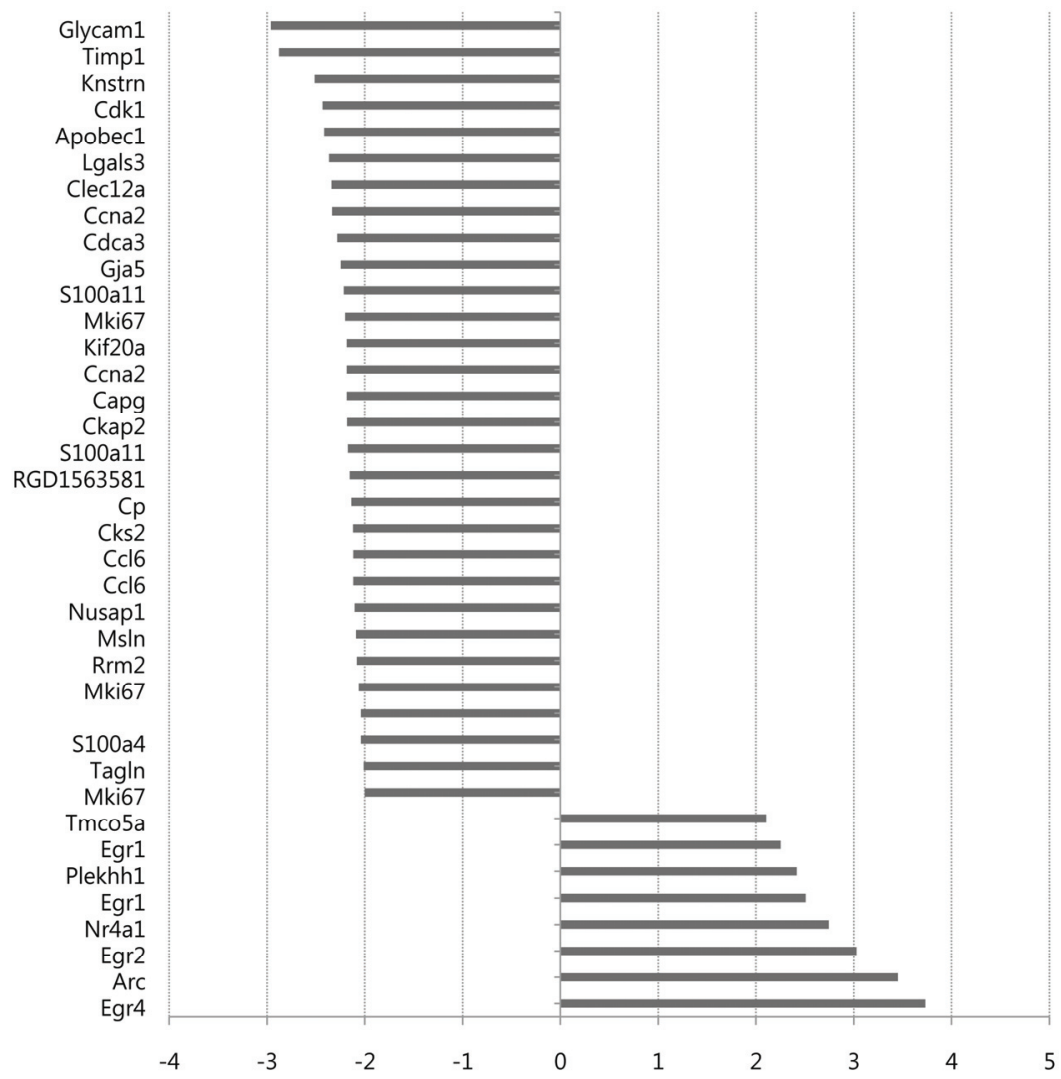

Fig. 2. List of DEGs with FD $\geq 2$ between the sham and MSC groups. The $\mathrm{x}$-axis represents the relative FD of gene expression between the MSC and sham groups. The full name of individual genes are described in Supplementary Table S2. attenuates the aberrant activation.

\section{Inflammation in the ischemic brain after IV-MSC}

As inflammation-related gene alterations are obvious following IV-MSC, we performed immunohistochemistry with the Iba-1 antibody to compare the extent of postischemic inflammation between the MSC $(n=4)$ and sham $(n=4)$ groups. In the sham group, numerous $\mathrm{lba}-1^{+}$cells were detected in peri-infarct areas of the ischemic brain $72 \mathrm{~h}$ after MCAo induction (Fig. 3A). In the MSC group, there were fewer $\mathrm{Iba}-1^{+}$cells than in the sham group $(37.9 \pm 2.2 \%$ vs. $49.8 \pm 3.2 \%$, respectively; $p=0.01$; Fig. $3 \mathrm{~B})$. These finding suggest that IV-MSC attenuates postischemic inflammation by reducing the number of activated immune cells in the ischemic brain.

\section{Infarct size and functional deficits in MCAo rats after IV-} MSC

We compared infarct sizes $72 \mathrm{~h}$ after MCAo using TTC staining. The mean infarct size was significantly smaller in the MSC group (MSC group vs. sham group: $29.4 \pm 1.9 \%$ vs. $37.1 \pm$ $1.4 \%, p=0.003$, both $n=7$; Fig. $4 \mathrm{~A}$ ).

We next compared behavioral deficits assessed with the mNSS test for 4 weeks between the MSC $(n=6)$ and sham ( =6) groups. Compared to sham animals, the MSC group showed a significant improvement in mNSS score from 14 to 28 days after MCAo (Fig. 4B). There was no difference in overall mortality between the two groups $(p=0.90)$. Our findings suggest that IV-MSC is neuroprotective during the acute phase of cerebral ischemia, which promotes subsequent functional recovery.

\section{DISCUSSION}

In the present study, we performed transcriptome analyses to investigate the therapeutic mechanism of IV-MSC in a rodent stroke model. The mRNA microarray results revealed that the expressions of numerous genes are changed in the postischemic brain, with most upregulated. Many dysregulated genes are related to inflammation and cell cycle. Notably, bioinformatics analysis revealed that IV-MSC attenuated the aberrant activation of immune response- and cell cyclerelated signaling pathways following ischemia. Consistent with previous microarray studies (Lu et al., 2003; RamosCejudo et al., 2012; Tang et al., 2002), we found that the expressions of numerous genes related to inflammation and the cell cycle were altered in the ischemic brain. In response to ischemia, microglia and peripheral macrophages are activated and release pro-inflammatory cytokines (e.g., interleukins and tumor necrosis factors) that upregulate the expression of adhesion molecules on endothelial cells. Leukocytes and platelets promote the transmigration of circulating immune cells into the brain parenchyma. Activated chemokines and their receptors recruit bone marrow-derived mononuclear cells and endothelial progenitors to the ischemic lesion. Our microarray results showed that cerebral ischemia altered the expression of numerous genes encoding cytokines (e.g., tumor necrosis factor [TNF], interleukin [IL]-18, etc.), cytokine receptors (e.g., TNF receptor superfamily, IL-4, IL-10, IL-13 receptors, etc.), adhesion molecules (intercellular adhesion molecule [ICAM], alpha and beta integrins, etc.), chemokines (e.g., chemokine [C-C motif] ligand [CCL]6, CCL9, CCL13, 
A

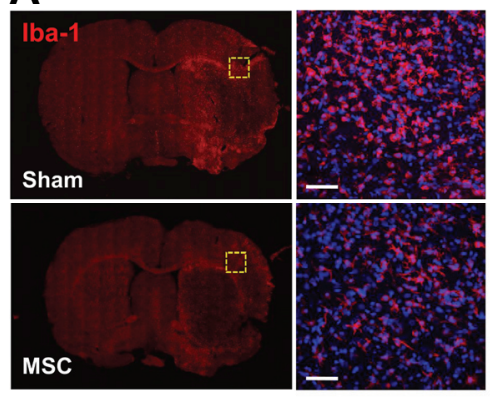

B

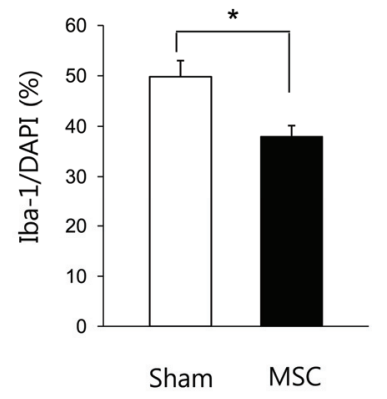

Fig. 3. Microglial activation changes in the postischemic brain after IV-MSC in MCAo rats. (A) Confocal microscopy for lba-1 immunohistochemistry in brain sections from the sham $(n=4)$ and MSC $(n=4)$ groups. The right panel represents the brain area indicated by the yellow dashed box in the left panel. Scale bar, $20 \mu \mathrm{m}$. A nuclear marker, 4', 6-diamidine-29phenylindole dihydrochloride (DAPI, blue color) was used for counterstaining. (B) Graph showing the percentage of $\mathrm{Iba}-1^{+}$ to $\mathrm{DAPI}^{+}$cells in the peri-infarct area of brains of the sham and MSC groups. ${ }^{*} p<0.05$ with Mann-Whitney $U$ test.
A

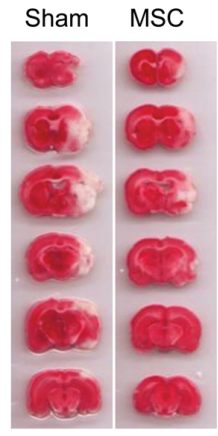

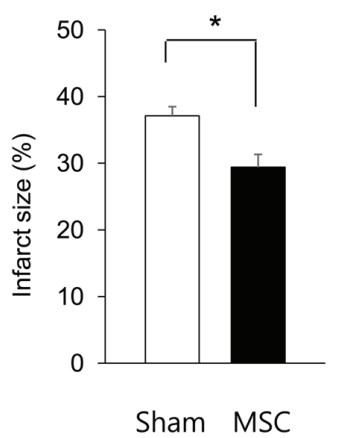

B

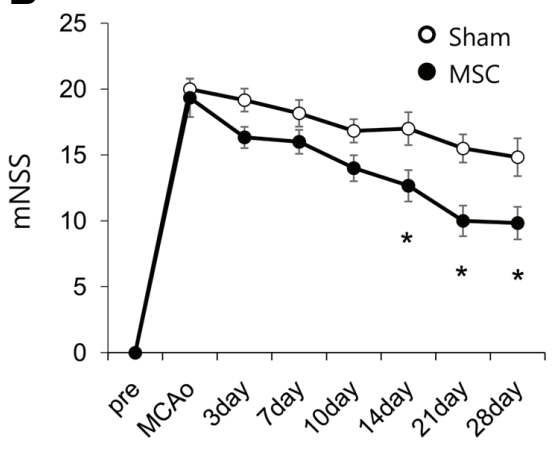

Fig. 4. Changes in infarct size and functional scores between the sham and MSC groups. (A) Measurement of infarct size $72 \mathrm{~h}$ after MCAo using 2,3,5triphenyltetrazolium chloride (TTC) staining in the sham $(n=7)$ and MSC $(n=7)$ groups. ${ }^{*} p<0.05$ with Mann-Whitney $U$ test. (B) mNSS scores between the sham $(n=6)$ and MSC $(n=6)$ groups. The 'pre' represents 1 day prior to MCAo induction. ${ }^{*} p<0.05$ with mixed ANOVA test. chemokine [C-X-C motif] ligand $[\mathrm{CXCL}] 10, \mathrm{C}-\mathrm{C}$ chemokine receptor [CCR]5, chemokine [C-X3-C motif] ligand 1 [Cx3CL1], etc.), and chemokine receptors (e.g., chemokine [C-X-C motif] receptor [CXCR]-4), pattern-recognition receptors [Toll-like receptor (TLR)]2, TLR4) and their signaling pathways (e.g., myeloid differentiation primary response 88 [MYD88], interferon regulatory factor 5 [IRF5], mitogen-activated protein kinase [MAPK], etc.) (Table 1), most of which have been associated with stroke-related neuroinflammation (ladecola and Anrather, 2011). The mRNA microarray findings showed that IV-MSC normalized the aberrant ischemia-induced expression of genes related to cytokines, chemokines, and their receptors. Furthermore, immunohistochemical analyses revealed that IV-MSC reduced the numbers of ${\mathrm{Iba}-1^{+}}^{+}$cells in the ischemic brain. Accumulating evidence suggests that MSCs have a strong immune-modulating property. MSCs attenuate postischemic inflammation in rats (Zhang et al., 2011) by reducing proinflammatory cytokine activation (e.g., IL-1, TNF- $\alpha$, or INF- $\gamma$ ) or enhancing anti-inflammatory cytokine expression (e.g., transforming growth factor-beta [TGF- $\beta$ ] and IL-10) (Eggenhofer and Hoogduijn, 2012; Lee et al., 2009; Nemeth et al., 2009). MSCs also induce macrophage phenotype in the milieu of excessive inflammation, with pro-inflammatory $\mathrm{M} 1$ subtype polarizing toward the anti-inflammatory M2 subtype (Bernardo and Fibbe, 2013). Based on the present findings, we propose that IV-MSC normalizes the aberrant expression of neuroinflammationrelated genes following cerebral ischemia.

Interestingly, the pathway analysis predicted that IV-MSC strongly ameliorated the antigen-mediated immune response in cerebral ischemia (e.g., SLE and Fc $\gamma R$-mediated phagocytosis signaling pathways). Cerebral ischemia activates both humoral and cell-mediated immunity in response to the presentation of tissue antigens such as myelin basic protein and related peptides that were previously veiled by the blood-brain barrier in the central nervous system (CNS). In response to CNS antigen presentation, circulating $\mathrm{T}$ lymphocytes become sensitized and infiltrate the ischemic brain, eventually leading to neuroinflammation (Arumugam et al., 2005; Ishikawa et al., 2005; Shichita et al., 2009; Yilmaz et al., 2006).

FcyRs are widely expressed in microglia in the CNS, (Vedeler et al., 1994) and recent studies have shown the pivotal role of $F c \gamma R$ in neuronal injury in an animal model of stroke. FcyR-/- mice exhibit reduced mortality and smaller infarct size, presumably due to less microglial activation and circulating lymphocyte infiltration compared to wild-type mice (KomineKobayashi et al., 2004). IV immunoglobulin markedly reduces mortality and infarct size by $50 \%$ in a rodent stroke model (Arumugam et al., 2007), and these effects are partially mediated by preventing Fc $\gamma \mathrm{R}$-mediated phagocytosis of antigen-bearing target cells and antibody-dependent cell mediated cytotoxicity (Bayry et al., 2007; Samuelsson et al., 2001). MSC reduces the antigen-mediated immune response by suppressing cytotoxic $T$ cells or enhancing the effect of regulatory $T$ cells through the release of prostaglandin E2 and indoleamine 2,3-dioxygenase in vitro (Aggarwal and Pittenger, 2005). The therapeutic effect of MSC on antigen-mediated immune response has been demonstrated in animal models of multiple sclerosis (Zappia et al., 2005), rheumatoid arthritis (Augello et al., 2007), graftversus-host diseases, and SLE (Sun et al., 2009). Although further studies are needed, our microarray analysis results suggest that attenuation of the antigen-mediated immune response in the ischemic brain is an important therapeutic mechanism of IV-MSC in the setting of cerebral ischemia.

Another interesting finding of our study is that cerebral is- 
Transcriptome Analysis in an IV-MSC-Treated Rat Stroke Model

Chunggab Choi et al.

Table 1. List of predicted signaling pathways for differentially expressed genes between the sham and normal groups

\begin{tabular}{|c|c|c|c|c|c|c|}
\hline Pathway & Count & $\%$ & $p$ & Corrected $p^{a}$ & FE & Genes \\
\hline $\begin{array}{l}\text { Systemic lupus } \\
\text { erythematosus }\end{array}$ & 17 & 2.55 & 5.51E-06 & 7.82E-04 & 3.85 & $\begin{array}{l}\text { Tnf, C6, Hist1h2ail, LOC498276, Hist2h3c2, RT1-DMa, C1r, C1s, } \\
\text { Cd40, RT1-DMb, C1qc, C1qa, C1qb, Fcgr2b, Hist2h2ac, } \\
\text { Fcgr1a, Fcgr2a, C2, Fcgr3a }\end{array}$ \\
\hline $\begin{array}{l}\text { Toll-like receptor } \\
\text { signaling pathway }\end{array}$ & 15 & 2.25 & 9.99E-05 & 7.07E-03 & 3.40 & $\begin{array}{l}\text { Ccl3, Tnf, Ly96, Tlr2, Tlr4, Mapk11, Cd40, Tlr6, Cxcl10, Fos, } \\
\text { Myd88, Irf5, Map3k8, Cd14, Spp1 }\end{array}$ \\
\hline $\begin{array}{l}\text { Complement and } \\
\text { coagulation cascades }\end{array}$ & 13 & 1.95 & $1.21 \mathrm{E}-04$ & 5.71E-03 & 3.79 & $\begin{array}{l}\text { A2m, C5ar1, C6, C1r, Serping1, C1s, C1qc, C1qa, Vwf, C1qb, } \\
\text { Cfh, C2, Cfd }\end{array}$ \\
\hline $\begin{array}{l}\text { Natural killer cell } \\
\text { mediated cytotoxicity }\end{array}$ & 15 & 2.25 & 3.14E-04 & $1.11 \mathrm{E}-02$ & 3.06 & $\begin{array}{l}\text { Icam1, Ptpn6, Tnf, Itgb2, Hcst, Rac2, Fcgr2b, Plgc2, Fcer1g, } \\
\text { Shc1, Fcgr2a, Fcgr3a, Ifngr1, Tyrobp, Lcp2, Syk }\end{array}$ \\
\hline Cell cycle & 17 & 2.55 & 3.73E-04 & 1.05E-02 & 2.75 & $\begin{array}{l}\text { LOC298795, Ttk, Pkmyt1, Cdk6, Chek1, Cdc20, Sfn, Mcm2, } \\
\text { Pttg1, Mcm3, Mcm4, Tgfb1, Mcm6, Ccnd1, Plk1, Bub1, Ccna2, } \\
\text { Myc }\end{array}$ \\
\hline $\begin{array}{l}\text { MAPK signaling } \\
\text { pathway }\end{array}$ & 27 & 4.05 & $4.88 \mathrm{E}-04$ & 1.15E-02 & 2.07 & $\begin{array}{l}\text { Tnf, Hspa1b, Nfkb2, Tgfb1, Map3k6, Tnfrsf1a, Fos, Bdnf, Rac2, } \\
\text { Rasgrp1, Rasgrp2, Map3k8, Rras, Myc, Fgf2, Ptpn7, Tgfbr2, } \\
\text { Nr4a1, Cacng3, Mapk11, Flna, Dusp5, Pla2g4a, Dusp1, Ntrk1, } \\
\text { Hspb1, Cd14 }\end{array}$ \\
\hline Focal adhesion & 21 & 3.15 & $1.22 \mathrm{E}-03$ & 2.44E-02 & 2.20 & $\begin{array}{l}\text { Parvg, Tln1, Col4a1, Col3a1, Igf1, Itga4, Birc3, Flna, Pak6, Vwf, } \\
\text { Ccnd1, Rac2, Vegfa, Col6a2, Rhoc, Shc1, Lamc1, Col11a2, } \\
\text { Parva, Spp1, Fn1 }\end{array}$ \\
\hline $\begin{array}{l}\text { Hematopoietic cell } \\
\text { lineage }\end{array}$ & 12 & 1.80 & $1.29 \mathrm{E}-03$ & 2.27E-02 & 3.14 & $\begin{array}{l}\text { Sept5, Tnf, Cd44, Cd8b, Fcgr1a, I14ra, Anpep, Itga4, Cd1d1, } \\
\text { Cd14, Csf2ra, Csf1r }\end{array}$ \\
\hline $\begin{array}{l}\text { Chemokine signaling } \\
\text { pathway }\end{array}$ & 19 & 2.85 & $1.58 \mathrm{E}-03$ & 2.47E-02 & 2.27 & $\begin{array}{l}\text { Adcy4, Ccl3, Fgr, Ncf1, Hck, Ccl9, Pf4, Cx3cl1, Stat3, Ccl6, } \\
\text { Cxcl10, Ccr5, Rac2, Cxcr4, Cxcl16, Rasgrp2, Rhoc, Shc1, } \\
\text { Jak3 }\end{array}$ \\
\hline $\begin{array}{l}\text { ECM-receptor } \\
\text { interaction }\end{array}$ & 12 & 1.80 & $1.77 \mathrm{E}-03$ & $2.48 \mathrm{E}-02$ & 3.02 & $\begin{array}{l}\text { Sept5, Vwf, Sdc1, Col4a1, Cd44, Col3a1, Col6a2, Lamc1, Itga4, } \\
\text { Col11a2, Fn1, Spp1 }\end{array}$ \\
\hline $\begin{array}{l}\text { Cytokine-cytokine } \\
\text { receptor interaction }\end{array}$ & 20 & 3.00 & $3.26 \mathrm{E}-03$ & 4.13E-02 & 2.07 & $\begin{array}{l}\text { Ccl3, Tnf, Tnfrsf12a, ॥18, ॥4ra, Tgfbr2, Pf4, Cd40, Cx3cl1, } \\
\text { Cxcl10, Tnfrsf1a, Tnfrsf1b, Ccr5, Cxcr4, Il10rb, Cxcl16, Il13ra1, } \\
\text { Csf2ra, Ifngr1, Csf1r }\end{array}$ \\
\hline $\begin{array}{l}\text { Leukocyte transendothelial } \\
\text { migration }\end{array}$ & 14 & 2.10 & 3.75E-03 & 4.35E-02 & 2.48 & $\begin{array}{l}\text { Icam1, Ncf1, Ncf4, Sipa1, Mapk11, Itgb2, Itga4, Cldn14, Cyba, } \\
\text { Rac2, Cxcr4, Plcg2, Rhoc, Msn }\end{array}$ \\
\hline Lysosome & 14 & 2.10 & 4.36E-03 & 4.66E-02 & 2.44 & $\begin{array}{l}\text { Tcirg1, Ctsz, Gusb, Lgmn, Napsa, Cd63, Ctsl1, Slc11a1, Ctsk, } \\
\text { Cd68, Laptm5, Ctsd, Ctsc, Cln5 }\end{array}$ \\
\hline
\end{tabular}

${ }^{a} p$-value after Benjamini-Hochberg correction

The full names of genes are described in Supplementary Table S1.

The genes italicized denote downregulated genes in the sham group compared to the normal group.

ECM, extracellular matrix; FE, fold enrichment; MAPK, mitogen-activated protein kinase

chemia alters the expression of cell cycle-related genes and these changes are ameliorated by IV-MSC. Previous reports support the critical role of cell cycle regulation in neuronal death following brain injury (Rashidian et al., 2007). Hypoxia induces the expression of major cell cycle regulators such as cyclin D1, cyclin-dependent kinase (Cdk) 2, and E2F transcription factor 1 (E2F1) in oxygen-glucose deprived neurons (Katchanov et al., 2001), implying that inappropriate activation of cell cycle machinery in differentiated neurons may elicit ischemic neuronal death. In an in vivo stroke model, expression levels of Cdk4 and cyclin D1 complex are altered following stroke (Rashidian et al., 2005), and treatment with their inhibitors has a neuroprotective effect in animal models of cerebral ischemia (Osuga et al., 2000; Wang et al., 2002). Our results suggest that IV-MSC attenuates the aberrant activation of cell cycle-related genes, thus preventing neuronal cell death. As a result, IV-MSC can reduce infarct size and promote functional improvement in a rodent stroke model.

Our study has several limitations, and further studies are needed to clarify the therapeutic mechanisms of IV-MSC in cerebral ischemia. Firstly, since our microarray analysis was conducted at a single time point (72 $\mathrm{h}$ after cerebral ischemia), we cannot reach a firm conclusion on therapeutic mechanisms of IV-MSC over time. As gene expression is dynamically altered at different time points after stroke, serial evaluation of gene expression changes is needed. Despite this shortcoming, our 
Table 2. List of predicted signaling pathways for differentially expressed genes between the MSC and sham groups

\begin{tabular}{lllllll}
\hline Pathway & Count & $\%$ & $p$ & Corrected $p^{\mathrm{a}}$ & FE & Genes \\
\hline Cell cycle & 10 & 5.68 & $1.60 \mathrm{E}-05$ & $1.23 \mathrm{E}-03$ & 6.52 & $\begin{array}{c}\text { Plk1, Bub1, Pkmyt1, Ttk, Cdc20, Pttg1, Mcm2, } \\
\text { Mcm3, Ccna2, Mcm6 }\end{array}$ \\
$\begin{array}{c}\text { Systemic lupus } \\
\text { erythematosus }\end{array}$ & 8 & 4.55 & $8.80 \mathrm{E}-05$ & $3.38 \mathrm{E}-03$ & 7.31 & $\begin{array}{c}\text { C1qb, Tnf, Fcgr2b, Fcgr1a, LOC498276, } \\
\text { Hist1h2ail, Fcgr3a, C1qc }\end{array}$ \\
$\begin{array}{c}\text { FcyR-mediated } \\
\text { phagocytosis }\end{array}$ & 7 & 3.98 & $5.93 \mathrm{E}-04$ & $1.51 \mathrm{E}-02$ & 6.54 & $\begin{array}{c}\text { Arpc1b, Fcgr2b, Rac2, Ncf1, Hck, Fcgr1a, } \\
\text { LOC498276 }\end{array}$ \\
\hline
\end{tabular}

${ }^{a} p$-value after Benjamini-Hochberg correction

The full names of genes are described in Supplementary Table S1.

The genes italicized denote downregulated genes in the MSC group compared to the sham group.

FE, fold enrichment; Fc $\gamma$ R, Fc-gamma receptor

results still provide valuable information regarding the molecular changes in response to ischemia and the effect of IV-MSCs at the acute phase $(72 \mathrm{~h})$ of cerebral ischemia, since brain injury is usually most extensive in the first several days after cerebral ischemia (ladecola and Anrather, 2011). Secondly, polymerase chain reaction-based analyses of individual DEGs should be performed to validate the microarray analysis results. Finally, our proposed therapeutic mechanism of IV-MSC in cerebral ischemia is dependent on bioinformatics analysis. We suggest that molecular changes following cerebral ischemia and IVMSC are not dependent on individual gene expression; rather, they are due to multiple, complex genomic alterations. The present study focused on the overall genomic pattern rather than the functional mechanisms of single genes.

In conclusion, transcriptome analyses revealed that IV-MSC attenuated the extent of genomic alterations in the postischemic brain. Amelioration of dysregulated inflammation- and cell cycle-related gene expression in the host brain underlies the beneficial effects of IV-MSC in cerebral ischemia. Our study provides valuable information regarding the molecular therapeutic mechanism of IV-MSC in cerebral ischemia.

Note: Supplementary information is available on the Molecules and Cells website (www.molcells.org).

\section{ACKNOWLEDGMENTS}

This study was supported by grants from the Basic Science Research Program (2011-0021344) through the National Research Foundation of Korea (NRF) funded by the Ministry of Science and Technology, the Next-Generation BioGreen 21 Program (No. PJ010002012014), the Rural Development Administration, and the Korea Healthcare Technology R\&D project (HC14C3297) of the Ministry for Health, Welfare \& Family Affairs, Republic of Korea.

\section{REFERENCES}

Aggarwal, S., and Pittenger, M.F. (2005). Human mesenchymal stem cells modulate allogeneic immune cell responses. Blood 105, 1815-1822.

Arumugam, T.V., Granger, D.N., and Mattson, M.P. (2005). Stroke and T-cells. Neuromol. Med. 7, 229-242.

Arumugam, T.V., Tang, S.C., Lathia, J.D., Cheng, A., Mughal, M.R., Chigurupati, S., Magnus, T., Chan, S.L., Jo, D.G., Ouyang, X., et al. (2007). Intravenous immunoglobulin (IVIG) protects the brain against experimental stroke by preventing complement-mediated neuronal cell death. Proc. Natl. Acad. Sci. USA 104, 1410414109.

Augello, A., Tasso, R., Negrini, S.M., Cancedda, R., and Pennesi,
G. (2007). Cell therapy using allogeneic bone marrow mesenchymal stem cells prevents tissue damage in collagen-induced arthritis. Arthritis. Rheum. 56, 1175-1186.

Bang, O.Y., Lee, J.S., Lee, P.H., and Lee, G. (2005). Autologous mesenchymal stem cell transplantation in stroke patients. Ann. Neurol. 57, 874-882.

Bayry, J., Lacroix-Desmazes, S., Kazatchkine, M.D., and Kaveri, S.V. (2007). Monoclonal antibody and intravenous immunoglobulin therapy for rheumatic diseases: rationale and mechanisms of action. Nat. Clin. Pract. Rheumatol. 3, 262-272.

Bernardo, M.E., and Fibbe, W.E. (2013). Mesenchymal stromal cells: sensors and switchers of inflammation. Cell Stem Cell 13, 392-402.

Chang, D.J., Lee, N., Park, I.H., Choi, C., Jeon, I., Kwon, J., Oh, S.H., Shin, D.A., Do, J.T., Lee, D.R., et al. (2013). Therapeutic potential of human induced pluripotent stem cells in experimental stroke. Cell Transplant. 22, 1427-1440.

Chen, J., Sanberg, P.R., Li, Y., Wang, L., Lu, M., Willing, A.E., Sanchez-Ramos, J., and Chopp, M. (2001). Intravenous administration of human umbilical cord blood reduces behavioral deficits after stroke in rats. Stroke 32, 2682-2688.

Deng, Y.B., Ye, W.B., Hu, Z.Z., Yan, Y., Wang, Y., Takon, B.F., Zhou, G.Q., and Zhou, Y.F. (2010). Intravenously administered BMSCs reduce neuronal apoptosis and promote neuronal proliferation through the release of VEGF after stroke in rats. Neurol. Res. 32, 148-156.

Eggenhofer, E., and Hoogduijn, M.J. (2012). Mesenchymal stem cell-educated macrophages. Transplant Res. 1, 12.

Eltzschig, H.K., and Eckle, T. (2011). Ischemia and reperfusion-from mechanism to translation. Nat. Med. 17, 1391-1401.

Honmou, O., Houkin, K., Matsunaga, T., Niitsu, Y., Ishiai, S., Onodera, R., Waxman, S.G., and Kocsis, J.D. (2011). Intravenous administration of auto serum-expanded autologous mesenchymal stem cells in stroke. Brain 134, 1790-1807.

ladecola, C., and Anrather, J. (2011). The immunology of stroke: from mechanisms to translation. Nat. Med. 17, 796-808.

Ikegame, Y., Yamashita, K., Hayashi, S., Mizuno, H., Tawada, M., You, F., Yamada, K., Tanaka, Y., Egashira, Y., Nakashima, S., et al. (2011). Comparison of mesenchymal stem cells from adipose tissue and bone marrow for ischemic stroke therapy. Cytotherapy 13, 675-685.

Ishikawa, M., Vowinkel, T., Stokes, K.Y., Arumugam, T.V., Yilmaz, G., Nanda, A., and Granger, D.N. (2005). CD40/CD40 ligand signaling in mouse cerebral microvasculature after focal ischemia/reperfusion. Circulation 111, 1690-1696.

Katchanov, J., Harms, C., Gertz, K., Hauck, L., Waeber, C., Hirt, L., Priller, J., von Harsdorf, R., Bruck, W., Hortnagl, H., et al. (2001). Mild cerebral ischemia induces loss of cyclin-dependent kinase inhibitors and activation of cell cycle machinery before delayed neuronal cell death. J. Neurosci. 21, 5045-5053.

Kim, J.M., Lee, S.T., Chu, K., Jung, K.H., Song, E.C., Kim, S.J., Sinn, D.I., Kim, J.H., Park, D.K., Kang, K.M., et al. (2007). Systemic transplantation of human adipose stem cells attenuated cerebral inflammation and degeneration in a hemorrhagic stroke model. Brain Res. 1183, 43-50.

Kim, S.M., Moon, S.H., Lee, Y., Kim, G.J., Chung, H.M., and Choi, 
Y.S. (2013). Alternative xeno-free biomaterials derived from human umbilical cord for the self-renewal ex-vivo expansion of mesenchymal stem cells. Stem Cells Dev. 22, 3025-3038.

Komine-Kobayashi, M., Chou, N., Mochizuki, H., Nakao, A., Mizuno, Y., and Urabe, T. (2004). Dual role of Fcgamma receptor in transient focal cerebral ischemia in mice. Stroke 35, 958-963.

Lee, R.H., Pulin, A.A., Seo, M.J., Kota, D.J., Ylostalo, J., Larson, B.L., Semprun-Prieto, L., Delafontaine, P,. and Prockop, D.J. (2009). Intravenous hMSCs improve myocardial infarction in mice because cells embolized in lung are activated to secrete the anti-inflammatory protein TSG-6. Cell Stem Cell 5, 54-63.

Lee, J.S., Hong, J.M., Moon, G.J., Lee, P.H., Ahn, Y.H., and Bang, O.Y. (2010). A long-term follow-up study of intravenous autologous mesenchymal stem cell transplantation in patients with ischemic stroke. Stem Cells 28, 1099-1106.

Liesz, A., Bauer, A., Hoheisel, J.D., and Veltkamp, R. (2014). Intracerebral interleukin-10 injection modulates post-ischemic neuroinflammation: an experimental microarray study. Neurosci. Lett. 579, 18-23.

Liu, X., Ye, R., Yan, T., Yu, S.P., Wei, L., Xu, G., Fan, X., Jiang, Y., Stetler, R.A., Liu, G., et al. (2014). Cell based therapies for ischemic stroke: from basic science to bedside. Prog. Neurobiol. $115,92-115$

Longa, E.Z., Weinstein, P.R., Carlson, S., and Cummins, R. (1989). Reversible middle cerebral artery occlusion without craniectomy in rats. Stroke 20, 84-91.

Lu, A., Tang, Y., Ran, R., Clark, J.F., Aronow, B.J., and Sharp, F.R. (2003). Genomics of the peri-infarction cortex after focal cerebral ischemia. J. Cereb. Blood Flow Metab. 23, 786-810.

Nemeth, K., Leelahavanichkul, A., Yuen, P.S., Mayer, B., Parmelee, A., Doi, K., Robey, P.G., Leelahavanichkul, K., Koller, B.H., Brown, J.M., et al. (2009). Bone marrow stromal cells attenuate sepsis via prostaglandin $E$ (2)-dependent reprogramming of host macrophages to increase their interleukin-10 production. Nat. Med. 15, $42-49$.

Osuga, H., Osuga, S., Wang, F., Fetni, R., Hogan, M.J., Slack, R.S., Hakim, A.M., Ikeda, J.E., and Park, D.S. (2000). Cyclindependent kinases as a therapeutic target for stroke. Proc. Natl. Acad. Sci. USA 97, 10254-10259.

Ramos-Cejudo, J., Gutierrez-Fernandez, M., Rodriguez-Frutos, B., Exposito Alcaide, M., Sanchez-Cabo, F., Dopazo, A., and DiezTejedor, E. (2012). Spatial and temporal gene expression differences in core and peri-infarct areas in experimental stroke: a microarray analysis. PLoS One 7, e52121.

Rashidian, J., Iyirhiaro, G., Aleyasin, H., Rios, M., Vincent, I., Callaghan, S., Bland, R.J., Slack, R.S., During, M.J., and Park, D.S. (2005). Multiple cyclin-dependent kinases signals are critical mediators of ischemia/hypoxic neuronal death in vitro and in vivo.
Proc. Natl. Acad. Sci. USA 102, 14080-14085.

Rashidian, J., Iyirhiaro, G.O., and Park, D.S. (2007). Cell cycle machinery and stroke. Biochim. Biophys. Acta 1772, 484-493.

Samuelsson, A., Towers, T.L., and Ravetch, J.V. (2001). Antiinflammatory activity of IVIG mediated through the inhibitory Fc receptor. Science 291, 484-486.

Shichita, T., Sugiyama, Y., Ooboshi, H., Sugimori, H., Nakagawa, R., Takada, I., Iwaki, T., Okada, Y., lida, M., Cua, D.J., et al. (2009). Pivotal role of cerebral interleukin-17-producing gammadelta $T$ cells in the delayed phase of ischemic brain injury. Nat. Med. 15, 946-950.

Slevin, M., Krupinski, J., Kumar, P., Gaffney, J., and Kumar, S. (2005). Gene activation and protein expression following ischaemic stroke: strategies towards neuroprotection. J. Cell. Mol. Med. 9, 85-102.

Sun, L., Akiyama, K., Zhang, H., Yamaza, T., Hou, Y., Zhao, S., Xu, T., Le, A., and Shi, S. (2009). Mesenchymal stem cell transplantation reverses multiorgan dysfunction in systemic lupus erythematosus mice and humans. Stem Cells 27, 1421-1432.

Tang, Y., Lu, A., Aronow, B.J., Wagner, K.R., and Sharp, F.R. (2002). Genomic responses of the brain to ischemic stroke, intracerebral haemorrhage, kainate seizures, hypoglycemia, and hypoxia. Eur. J. Neurosci. 15, 1937-1952.

Vedeler, C., Ulvestad, E., Grundt, I., Conti, G., Nyland, H., Matre, R., and Pleasure, D. (1994). Fc receptor for IgG (FcR) on rat microglia. J. Neuroimmunol. 49, 19-24.

Walczak, P., Zhang, J., Gilad, A.A., Kedziorek, D.A., Ruiz-Cabello, J., Young, R.G., Pittenger, M.F., van Zijl, P.C., Huang, J., and Bulte, J.W. (2008). Dual-modality monitoring of targeted intraarterial delivery of mesenchymal stem cells after transient ischemia. Stroke 39, 1569-1574.

Wang, F., Corbett, D., Osuga, H., Osuga, S., Ikeda, J.E., Slack, R.S., Hogan, M.J., Hakim, A.M., and Park, D.S. (2002). Inhibition of cyclin-dependent kinases improves CA1 neuronal survival and behavioral performance after global ischemia in the rat. J. Cereb. Blood Flow Metab. 22, 171-182.

Yilmaz, G., Arumugam, T.V., Stokes, K.Y., and Granger, D.N. (2006). Role of T lymphocytes and interferon-gamma in ischemic stroke. Circulation 113, 2105-2112.

Zappia, E., Casazza, S., Pedemonte, E., Benvenuto, F., Bonanni, I. Gerdoni, E., Giunti, D., Ceravolo, A., Cazzanti, F., Frassoni, F., et al. (2005). Mesenchymal stem cells ameliorate experimental autoimmune encephalomyelitis inducing T-cell anergy. Blood 106, 1755-1761.

Zhang, L., Li, Y., Zhang, C., Chopp, M., Gosiewska, A., and Hong, K. (2011). Delayed administration of human umbilical tissuederived cells improved neurological functional recovery in a rodent model of focal ischemia. Stroke 42, 1437-1444. 